\section{Quand le squelette des cellules produit des forces}

Martin Lenz
LPTMS, CNRS,

Université Paris-Sud, Université Paris-Saclay, 15, rue Georges Clémenceau, 91405 Orsay, France. martin.lenz@u-psud.fr
> Nos cellules sont loin de n'être que des sacs de protéines jouant le rôle de réacteurs biochimiques. Nombre d'entre elles ont ainsi la faculté de se mouvoir et de se déformer de leur propre chef, une fonction essentielle pour des processus aussi divers que la division cellulaire, le façonnement de l'embryon ou encore la contraction musculaire [1]. Tous ces comportements ont cependant la même origine microscopique : les forces qu'ils impliquent sont produites par l'action simultanée d'une multitude de moteurs moléculaires contenus à l'intérieur de la cellule. Ces forces sont ensuite transmises pour passer de l'échelle nanométrique du moteur moléculaire - la myosine - à celle de la cellule, mille fois plus grande, via le cytosquelette d'actine, un assemblage de fibres protéiques souples.

Alors que l'attention des chercheurs a longtemps été focalisée sur le fonctionnement des moteurs individuels et les mécanismes biophysiques à l'œuvre au niveau d'un seul filament d'actine, il devient de plus en plus clair que l'interaction entre de nombreux moteurs et filaments peut avoir des conséquences qu'il aurait été difficile d'imaginer en les examinant un à un. Pour faire ce lien entre le microscopique et le macroscopique, la biologie bénéficie grandement de l'apport de la science des matériaux et de la mécanique statistique, domaine de la physique qui examine les comportements collectifs émergents de grands nombres de particules [2].

\section{L'énigme de la contraction désordonnée}

La biologie du cytosquelette a connu des progrès spectaculaires au cours des

dernières décennies et ses composants moléculaires sont maintenant largement caractérisés. Passant sous silence la myriade de protéines associées nécessaires à leur bon fonctionnement, ses deux acteurs principaux sont la myosine [3] $(\rightarrow)$, un moteur moléculaire

$(\rightarrow)$ Voir la Nouvelle de J. Ménétrey et al., $\mathrm{m} / \mathrm{s} \mathrm{n}^{\circ} 2$, février 2006, page 120 dont les isoformes les plus courantes s'associent sous forme de grappes, et l'actine, un polymère semi-rigide dont les constituants s'associent têteà-queue pour former un filament à la polarité bien déterminée. Cette polarité est essentielle pour l'action de la myosine: une grappe de moteurs associée à un filament aura toujours tendance à «marcher» le long du filament pour se diriger vers son bout dit barbé en s'éloignant du bout dit pointu. Cette simple propriété permet de comprendre la contraction de nos muscles striés, structures très bien organisées où les moteurs sont systématiquement localisés à proximité du bout pointu de l'actine. Lorsque les moteurs y sont activés, ils tirent à eux l'actine et induisent ainsi la contraction, comme l'illustre la Figure IA.

Bien que prédominants dans la production de nos mouvements volontaires, nos muscles striés ne sont qu'une des nombreuses structures à même d'induire la contraction au sein de nos cellules. Dans de nombreux autres assemblages, tels que le cortex d'actine bi-dimensionnel qui sous-tend la membrane de nos cellules, l'agencement des filaments d'actine est largement désordonné [4]. $\varepsilon$ n raison de cet agencement désordonné, l'origine de la contraction y est beaucoup moins évidente. $\varepsilon$ n effet, si certains des moteurs de myosine sont situés à proximité des bouts pointus de l'actine, et engendrent donc la contraction comme dans les muscles striés, il est tout aussi probable de trouver des moteurs à proximité des bouts barbés (Figure 1B). En vertu des règles décrites ci-dessus, de tels moteurs engendrent l'extension locale du cytosquelette. Ainsi que nous l'avons récemment montré par des arguments théoriques [5], il est donc impossible d'expliquer la contractilité du cytosquelette désordonné par les seuls arguments habituellement utilisés pour discuter des muscles striés.

\section{Les filaments prennent le centre de la} scène

Afin de proposer un mécanisme pour l'émergence de cette contractilité, nous nous sommes intéressés à la transmission des forces des moteurs par le réseau d'actine qui les entoure [6]. Plutôt que de chercher l'origine de la contraction dans le fonctionnement des moteurs eux-mêmes, nous avons admis qu'ils induisent localement autant de contraction que d'extension. Nous nous sommes en revanche interrogés sur le devenir de ces forces de signes opposés lors de leur transmission par les filaments environnants : une situation où la contraction et l'extension sont présentes en quantités égales au niveau microscopique de la protéine pourrait-elle ainsi donner lieu à une contraction au niveau macroscopique de la cellule?

Pour aborder ce point de principe, nous avons mené une étude purement théorique, fondée en partie sur des 

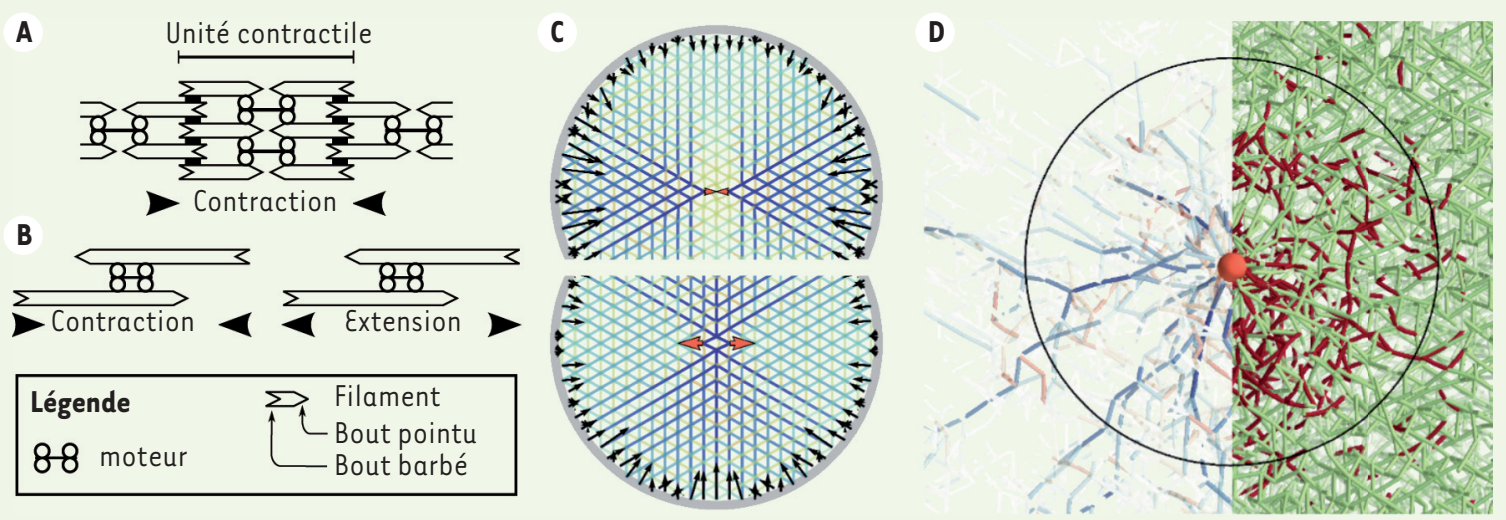

Figure 1. Production de force par les moteurs et transmission par les filaments d'actine. A. Nos muscles striés sont organisés en unités contractiles (les sarcomères) périodiques, où les moteurs sont localisés auprès des bouts pointus alors que les bouts barbés sont attachés ensemble par des protéines réticulantes (rectangles noirs). B. Des structures dans lesquelles cette organisation de polarité n'est pas présente peuvent engendrer des forces contractiles aussi bien qu'extensiles. C. Simulations numériques dans lesquelles les forces engendrées par un petit moteur contractile (flèches oranges, en haut) ou extensile (en bas) sont transmises par un réseau dont la couleur indique la tension de ses filaments (bleu : tension ; rouge : compression). Les forces au bord résultantes, toutes contractiles, sont représentées par des flèches noires. $D$. Réagencement des filaments d'un réseau tri-dimensionnel désordonné sous l'effet des forces du moteur (sphère orange). La moitié gauche de l'image met en valeur des chaînes de force très tendues (bleu), alors que, sur la moitié droite, les filaments flambés sont représentés en rouge. Le cercle noir figure la zone où le flambage est prédominant. Les panneaux $C$ et $D$ sont adaptés de arXiv:1507.05873 (https://arxiv.org/abs/1507.05873). (C Creative Commons Attribution license (https://arxiv.org/help/license) (https://creativecommons.org/licenses/by/4.0/).

simulations numériques. Nous avons ainsi programmé dans un ordinateur les caractéristiques mécaniques d'un réseau de filament réticulé. Ce réseau est similaire au cortex d'actine, mais aussi aux réseaux lamellaires [7], présents dans les kératocytes motiles ou dans la matrice extracellulaire de tissus conjonctifs, au sein de laquelle des cellules entières exercent des forces. Une caractéristique cruciale de ces filaments est leur capacité à «flamber » lorsqu'ils sont mis sous compression. Derrière ce mot se cache un comportement mécanique fort familier pour qui a un jour manipulé un morceau de ficelle ou une tige de plastique souple: tout comme les filaments d'actine, ces objets résistent lorsque I'on tire dessus, mais cèdent et se courbent lorsqu'ils sont mis sous compression. Nous avons ensuite introduit dans notre réseau «flambable» des moteurs (virtuels) exerçant des forces localement contractiles ou extensiles, et nous avons mesuré les forces que le réseau lui-même exerce sur son envi- ronnement, matérialisé par une bordure circulaire rigide. Le résultat de cette expérience est frappant: quelles que soient les caractéristiques géométriques du moteur, son effet à grande distance se trouve toujours être d'engendrer la contraction dans toutes les directions, ainsi que nous l'illustrons dans la Figure 1C. Nous avons en effet observé que tout moteur, même extensile, exerce toujours une petite quantité de forces tensiles sur son voisinage. En raison du flambage des filaments environnants, ces forces sont les seules à pouvoir se propager au sein du réseau, ce qui pourrait expliquer la prédominance de la contraction dans les systèmes de filaments semiflexibles présents dans nos cellules.

\section{La mollesse fait la force}

Au-delà de cet effet qualitatif, nous nous sommes interrogés sur l'amplitude des forces contractiles résultant de ce mécanisme. Là encore, les résultats défient l'intuition. En raison du flambage des filaments, le réseau à proximité du moteur est réorganisé sous forme d'un agencement radial de chaînes de force sous forte tension. Ces chaînes n'amplifient pas en ellesmêmes la force du moteur, mais la transmettent sur des distances bien plus grandes qu'en l'absence de flambage (Figure ID). En conséquence, la contrainte mécanique résultante au niveau de l'ensemble du réseau s'en trouve amplifiée d'un facteur qui peut, en principe, être arbitrairement grand. Un obstacle s'oppose cependant à la production de forces potentiellement colossales: lorsque les chaînes de force rencontrent le bord du système, ou bien les chaînes induites par un autre moteur, elles cessent de s'allonger et l'amplification sature.

$\varepsilon$ n pratique, nos résultats théoriques nous ont encouragés à mettre en relation des données expérimentales disparates, sur les forces induites par un moteur seul d'une part, et sur les forces induites par un réseau complet comprenant un grand nombre de myosines d'autre part. Ces résultats peuvent aussi s'appliquer à 
des cellules entières plongées dans une matrice cellulaire flexible. Nous avons ainsi pu comparer les forces engendrées par une plaquette seule aux contraintes contractiles que peut exercer un caillot sanguin contenant un grand nombre de ces plaquettes. Dans les deux cas, les forces générées collectivement par le réseau entier apparaissent hors de proportion avec une prédiction naïve qui ignorerait le flambage, et notre théorie rend compte avec une bonne précision d'amplifications pouvant aller jusqu'à un facteur vingt.

Au-delà de simples mesures de force, nos résultats sont en bon accord avec l'observation directe de la contraction de systèmes d'actomyosine reconstruits à partir de protéines purifiées, dans lesquels le flambage est prépondérant [8]. La question de l'applicabilité de ces mécanismes physiques simples au sein de la cellule reste cependant ouverte. 0 n est par exemple en droit de se demander si les filaments d'actine y flambent vraiment sous compression, ou s'ils sont coupés par des protéines spécialisées comme la cofiline ${ }^{1}$, dont l'action semble dépendre de la tension du filament [9]. Une chose reste cependant certaine: si elle n'apporte pas toutes les réponses, la physique et ses raisonnements géométriques et quantitatifs permettent de déceler des questions latentes au sein de mécanismes que l'on croyait bien compris. $\diamond$

When the cell skeleton produces forces

\section{LIENS D'INTÉRÊT}

L'auteur déclare n'avoir aucun lien d'intérêt concernant les données publiées dans cet article.

${ }^{1}$ Protéine qui déstabilise les filaments d'actine.

\section{RÉFÉRENCES}

1. Blanchoin L, Boujemaa-Paterski R, Sykes C, Plastino J. Actin dynamics, architecture, and mechanics in cell motility. Physiol Rev 2014 ; 94 : 235-63.

2. Murrell M, Oakes PW, Lenz M, Gardel ML. Forcing cells into shape: the mechanics of actomyosin contractility. Nat Rev Mol Cell Biol 2015 ; 16 : 486-98.

3. Ménétrey J, Bahloul A, Houdusse A. Une myosine à contre-sens. Med Sci (Paris) 2006 ; 22 : 120-2.

4. Medalia 0 , Weber I, Frangakis AS, et al. Macromolecular architecture in eukaryotic cells visualized by cryoelectron tomography. Science 2002 ; 298: 1209-13.

5. Ronceray P, Lenz M. Connecting local active forces to macroscopic stress in elastic media. Soft Matter 2015 ; 11 : 1597-605.

6. Ronceray P, Broedersz C, Lenz M. Fiber networks amplify active stress. Proc Natl Acad Sci USA 2016 ; $113: 2827-32$.

7. Verkhovsky AB, Svitkina TM, Borisy GG. Myosin Il filament assemblies in the active lamella of fibroblasts: their morphogenesis and role in the formation of actin filament bundles. J Cell Biol 1995 ; $131:$ 989-1002.

8. Murrell MP, Gardel ML. F-actin buckling coordinates contractility and severing in a biomimetic actomyosin cortex. Proc Natl Acad Sci USA 2012 ; 109 : 20820-5.

9. Hayakawa K, Tatsumi H, Sokabe M. Actin filaments function as a tension sensor by tension-dependent binding of cofilin to the filament. J Cell Biol 2011 ; 195: 721-7.

\section{NOUVELLE}

\section{Les règles de la biodiversité en temps profond}

Éric Lewitus, Hélène Morlon
Institut de Biologie, દ́cole Normale

Supérieure, 46, rue d'Ulm,

75005 Paris, France.

lewitus@biologie.ens.fr

mais aussi leur temps de divergence (Figure 1A). Les phylogénies moléculaires datées ouvrent une fenêtre sur l'évolution de la diversité de la vie et sont nos guides les plus puissants pour comprendre comment les espèces ont évolué. Elles sont utilisées, par exemple, pour étudier l'évolution de vecteurs de maladies infectieuses [2], les réponses de la diversité biologique aux changements environnementaux [3], ou encore la diversification des traits des espèces qui leur confère leur rôle unique [4]. Les arbres phylogénétiques sont un enregistrement des événements de spéciaon peut reconstruire non seulement la relation de parenté entre les espèces

\section{L'évolution vue à travers l'arbre du}

La vie sur Terre est étonnamment diversifiée. On estime à plus de dix millions le un rôle unique dans les écosystèmes de notre planète. Ces espèces descendent sont apparues par accumulation des processus de mutation et spéciation. L'arbre du vivant (ou phylogénie) est une représentation du lien de parenté 